\title{
Development of Path Loss Model for 802.11n in Large Conference Rooms
}

\author{
F. Heereman*, W. Joseph, E. Tanghe, D. Plets and L. Martens \\ Department of Information Technology \\ Ghent University/IBBT \\ Ghent, Belgium \\ Email: frederic.heereman@intec.ugent.be
}

\begin{abstract}
In this paper, a path loss (PL) model for 802.11n in large conference rooms is determined, based on PL measurements. The PL can be described accurately by a one-slope model with one standard deviation. PL exponents varying from 1.2 to 1.7 are found. Based on this PL model, the effect of frequency (2.4 vs $5 \mathrm{GHz}$ ), configuration (SISO vs MIMO (spatial diversity)), bandwidth (20 vs $40 \mathrm{MHz})$ and transmit power on number of access points, total power consumption and possible (physical) throughputs is investigated. According to the determined PL model, a higher range (by tuning the transmit power) requires less access points, as well as a lower total power consumption, due to a PL exponent lower than 2.
\end{abstract}

\section{INTRODUCTION}

The Wireless LAN Standard IEEE 802.11n, released in 2009 , is an amendment to the previous standards 802.11a and $802.11 \mathrm{~g}$ to provide higher throughputs [1]. Modifications to the physical layer comprise MIMO (Multiple-Input MultipleOutput), the $2.4 / 5 \mathrm{GHz}$ band and a bandwidth of 20 or $40 \mathrm{MHz}$. Video streaming in large conference rooms, such as the European Parliament, requires throughputs of $55 \mathrm{Mbps}$ (up to 24 video channels) and more. 802.11n might be suitable for this application.

In literature, no much path loss (PL) models can be found specifically for large conference rooms. The IEEE 802.11 TGn channel model could be applicable [2]. However, this model applies to very different types of environment (from residential to large space (indoors - outdoors)), and possibly does not take into account the specific geometry of large conference rooms (e.g. hemicycles). In this paper, a PL model for large conference rooms is determined, based on PL measurements. This model will be compared with the TGn channel model.

Based on this PL model, the effect of typical 802.11n features (including frequency, bandwidth and MIMO configuration) on number of access points, total power consumption and possible (physical) throughputs will be investigated, with the focus on large conference rooms. This evaluation will be compared again with the TGn channel model.

\section{PATH LOSS MEASUREMENTS}

The path loss measurements were carried out in a large conference room in the European Parliament in Brussels. This room has a hemicycle geometry and contains about 350 seats (Fig. 1). The measurements were done at frequencies 2.4 and $5.4 \mathrm{GHz}$, corresponding to the 2 bands of $802.11 \mathrm{n}$.

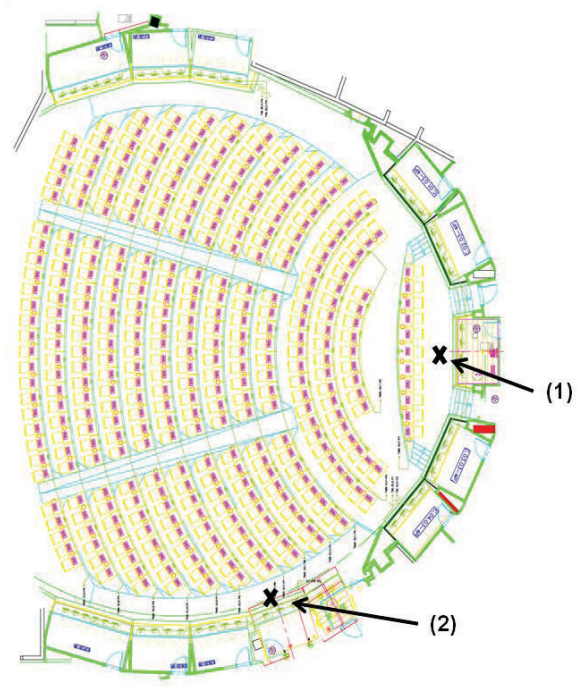

Fig. 1. Plan of conference room in European Parliament (Brussels), where PL measurements were carried out. (Plan taken over from Televic)

We considered 2 transmitter (Tx) positions. The first one is near the centre of the hemicycle ((1) in Fig. 1), at a height of $2 \mathrm{~m}$ and at a distance of $1 \mathrm{~m}$ from the wall. The second position is at the side of the room ((2) in Fig. 1), at a height of $3.5 \mathrm{~m}$ and about $10 \mathrm{~cm}$ from the wall. The Tx positions were chosen to get a LOS condition for all the seats. The receiver $(\mathrm{Rx})$ was positioned just above the desks (i.e. the actual position of the clients). The measured trajectories, which the receiver moved along, included all rows of desks.

As measurement equipment at the Tx side, we used the Rohde\&Schwarz signal generator SMJ100A, connected to a transmitting antenna. The equipment at the Rx side included a receiving antenna, connected to the Hewlett Packard spectrumanalyzer $8561 \mathrm{~B}$, and a tachometer. The spectrumanalyzer and the tachometer were connected to a laptop, which saved the received power and the distance along the $\mathrm{Rx}$ trajectory as a function of time. We used the omnidirectional MATJAYBEAM antenna MA431Z00 for $2.4 \mathrm{GHz}$, and the European Antennas antenna EVD2-5300/1285 for $5.4 \mathrm{GHz}$.

During the measurements, there was no people in the room. Consequently, these measurements allow to determine a PL 
model (including shadowing), but no temporal fading.

\section{PATH LOSS MODEL}

From the measurement data, we calculate the path loss $[\mathrm{dB}]$ by

$$
P L=-\left\langle P_{R}\right\rangle+P_{T}+G_{T}+G_{R}-L_{T}-L_{R},
$$

where $\left\langle P_{R}\right\rangle$ is the averaged received power $\left(\mathrm{P}_{\mathrm{R}}\right)[\mathrm{dBm}], \mathrm{P}_{\mathrm{T}}$ is the transmit power $[\mathrm{dBm}], \mathrm{G}_{\mathrm{T}}\left(\mathrm{G}_{\mathrm{R}}\right)$ is the transmitter (receiver) gain [dBi], and $\mathrm{L}_{\mathrm{T}}\left(\mathrm{L}_{\mathrm{R}}\right)$ is the transmitter (receiver) feeder loss [dB].

From the measurement data, we get the $\mathrm{P}_{\mathrm{R}}$ samples and their corresponding position (distance along measured trajectory). To calculate $\left\langle P_{R}\right\rangle$, we average the lineair interpolation of the $\mathrm{P}_{\mathrm{R}}$ samples over a distance of $10 \lambda$, where $\lambda$ is the wavelength.

During the measurements, we used a transmit power of $15 \mathrm{dBm}$. We determined experimentally the feeder losses: $\mathrm{L}_{\mathrm{T}}$ is $4.1 \mathrm{~dB}$ at $2.4 \mathrm{GHz}$ and $7.6 \mathrm{~dB}$ at $5.4 \mathrm{GHz} ; \mathrm{L}_{\mathrm{R}}$ is $2.2 \mathrm{~dB}$ at $2.4 \mathrm{GHz}$ and $3.5 \mathrm{~dB}$ at $5.4 \mathrm{GHz}$.

We determine the gain $(\mathrm{G})$ of transmitter and receiver as follows:

$$
G=G_{\max }+F(\theta),
$$

where $\mathrm{G}_{\max }$ is the gain [dBi] in the horizontal plane, and $\mathrm{F}$, defined by $\mathrm{G}-\mathrm{G}_{\max }$, depends on $\theta$, the angle with the horizontal axis. It is necessary to consider an angle-dependent gain, since there are angles $\theta$ up to $47^{\circ}$, and the $3 \mathrm{~dB}$ beamwidth is $40^{\circ}$ and $80^{\circ}$ for the $2.4 \mathrm{GHz}$ and $5.4 \mathrm{GHz}$ antenna respectively. For the antennas used at $2.4 \mathrm{GHz}$, we use $\mathrm{G}_{\max }$ and $F(\theta)$ from the datasheet. For the antennas used at $5.4 \mathrm{GHz}$, we know $\mathrm{G}_{\max }$ from the datasheet, but have no data for $\mathrm{F}(\theta)$. Therefore, we determine $\mathrm{F}$ by a theoretical approximation, applying to thin wire antennas, proposed in [3]:

$$
F=10 \log \left(\left(\frac{\cos (k L \sin (\theta))-\cos (k L)}{\cos (\theta)(1-\cos (k L))}\right)^{2}\right)
$$

where $\mathrm{k}=2 \pi / \lambda$, and $2 \mathrm{~L}$ is the length of the antenna. The $3 \mathrm{~dB}$ bandwidth (given in the datasheet) allows to determine the parameter $\mathrm{kL}$ in equation $3: \mathrm{kL}=1.426$.

We determine PL models for the different cases ( 2 frequencies, 2 Tx positions), based on PL samples calculated with equation 1 , in positions (along the trajectory) with a separation of $\lambda / 40$. We describe the path loss [dB] versus distance $\mathrm{d}[\mathrm{m}]$ between $\mathrm{Tx}$ and Rx by a one-slope model, with one standard deviation $\sigma[\mathrm{dB}]$ :

$$
P L=P L_{0}+10 n \log (d),
$$

where $\mathrm{PL}_{0}$ is the path loss at a distance of $1 \mathrm{~m}$, and $\mathrm{n}$ is the PL exponent. The parameters $\mathrm{PL}_{0}$ and $\mathrm{n}$, determined by the method of least squares, are shown in Table I, as well as the region where the PL could be experimentally determined. The determined PL exponents vary from 1.2 to 1.7 , which is lower than the free space PL exponent of 2.

For all cases, we found that it is possible to describe the path loss accurately by a one-slope model with one
TABLE I

PARAMETERS OF PL MODEL, BASED ON PL MEASUREMENTS IN A LARGE CONFERENCE ROOM.

\begin{tabular}{|c|c|c|c|c|c|c|}
\hline frequency & $\begin{array}{c}\mathrm{Tx} \\
\text { position }\end{array}$ & $\mathrm{n}$ & $\begin{array}{c}\mathrm{PL}_{0} \\
{[\mathrm{~dB}]}\end{array}$ & $\begin{array}{c}\mathrm{d}_{\mathrm{br}} \\
{[\mathrm{m}]}\end{array}$ & $\begin{array}{c}\sigma \\
{[\mathrm{dB}]}\end{array}$ & $\begin{array}{c}\text { considered } \\
\text { region }\end{array}$ \\
\hline $2.4 \mathrm{GHz}$ & front & 1.4 & 43 & 3.9 & 2 & $5-24 \mathrm{~m}$ \\
\cline { 2 - 7 } & side & 1.7 & 40 & 1.2 & 2 & $5-26 \mathrm{~m}$ \\
\hline \multirow{2}{*}{$5.4 \mathrm{GHz}$} & front & 1.2 & 51 & 3.0 & 2 & $5-24 \mathrm{~m}$ \\
\cline { 2 - 7 } & side & 1.2 & 53 & 4.9 & 2 & $5-27 \mathrm{~m}$ \\
\hline
\end{tabular}

standard deviation. This is illustrated in Fig. 2, where local percentiles, based on PL samples from a local region of $4 \mathrm{~m}$, are shown. The median can be modeled by a one-slope model, with a deviation less than $1 \mathrm{~dB}$. The shift between the 75 th percentile and the median is almost constant, which suggests one standard deviation.

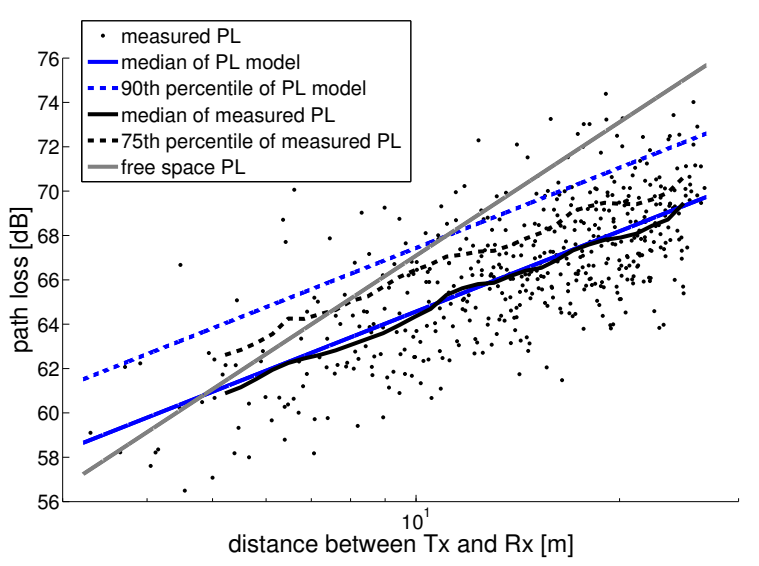

Fig. 2. Measured PL and PL model in large conference room (at $5.4 \mathrm{GHz}$, Tx position at the side). Percentiles based on the measured PL samples show that the PL can be described accurately by a one-slope model with one standard deviation. For purpose of review, only PL samples of positions separated by $10 \lambda$ are shown.

It is usefull to express the PL model by

$$
P L=P L_{f r e e, 0}+10 n \log \left(d / d_{b r}\right)
$$

where breakpoint $\mathrm{d}_{\mathrm{br}}$ is the distance [m] between Tx and Rx where the one-slope model intersects with the free space path loss, and $\mathrm{PL}_{\text {free }, 0}$ is the free space path loss $[\mathrm{dB}]$ at distance $\mathrm{d}_{\mathrm{br}}$. The corresponding breakpoint parameters, shown in Table $\mathrm{I}$, vary from 1 to $5 \mathrm{~m}$.

According to the IEEE 802.11 TGn channel model [2], the PL can be modeled by the free space PL for $\mathrm{d}<\mathrm{d}_{\mathrm{br}}$, and by a one-slope model with exponent 3.5 for $d>d_{b r}$. The TGn model predicts a breakpoint of $20 \mathrm{~m}$ for large office and $30 \mathrm{~m}$ for large space (indoors - outdoors). Compared to the TGn channel model, the PL model, determined for a large conference room, has a lower breakpoint and a lower PL exponent for $d>d_{b r}$. This results in a much lower PL. 


\section{RANGE, NUMBER OF ACCESS POINTS AND TOTAL POWER CONSUMPTION}

Based on the determined PL model, the range $\mathrm{R}$ [m] can be calculated by the link budget relation:

$P_{T}-P_{\text {sens }}+G_{T}+G_{R}-L_{T}-L_{R}=P L(R)+M_{S}+M_{F}$,

where $P_{\text {sens }}$ is the receiver sensitivity $[\mathrm{dBm}], \mathrm{PL}(\mathrm{d})$ is the PL model $[\mathrm{dB}]$ versus distance $\mathrm{d}$ between transmitter and receiver, $\mathrm{M}_{\mathrm{S}}$ is the shadowing margin $[\mathrm{dB}]$ and $\mathrm{M}_{\mathrm{F}}$ is the temporal fading margin $[\mathrm{dB}]$.

We estimate the number of access points (\#AP) as

$$
\# A P=S /\left(\pi R^{2}\right),
$$

where $S\left[\mathrm{~m}^{2}\right]$ is the area of the room. The power consumption $\mathrm{P}[\mathrm{W}]$ is calculated as

$$
P=\# A P P_{T},
$$

where $\mathrm{P}_{\mathrm{T}}$ is the transmit power [W].

Equations 6 to 8 allow to investigate the influence of the frequency $(2.4 / 5 \mathrm{GHz}$ band), configuration (SISO (SingleInput Single-Output)/MIMO $2 \times 2)$, bandwidth $(20 / 40 \mathrm{MHz})$ and transmit power on the required number of access points, total power consumption and maximum physical throughput $\left(\mathrm{TP}_{\max }\right)$.

For this calculation, receiver sensitivities of the 'reference' receiver from [1] are used. Compared to SISO, the sensitivities are decreased by $n_{T} \cdot n_{R}[d B]$ for MIMO, where $n_{T}$ is the number of antenna elements of the transmitter, and $n_{R}$ is the number of antenna elements of the receiver. Compared to a bandwidth of $20 \mathrm{MHz}$, the sensitivities are increased by $3 \mathrm{~dB}$ for $40 \mathrm{MHz}$.

Figs. 3 to 5 show the calculated range, number of access points and total power consumption vs transmit power for a sensitivity of $-64 \mathrm{dBm}$ (for the 'reference' receiver with a SISO configuration, a bandwidth of $20 \mathrm{MHz}$, Modulation \& Coding Scheme (MCS) 7), $\mathrm{G}_{\mathrm{T}}=2 \mathrm{dBi}, \mathrm{G}_{\mathrm{R}}=2 \mathrm{dBi}, \mathrm{L}_{\mathrm{T}}=0 \mathrm{~dB}, \mathrm{~L}_{\mathrm{R}}=$ $0 \mathrm{~dB}$ and $\mathrm{M}_{\mathrm{F}}=5.8 \mathrm{~dB}$. The margin $\mathrm{M}_{\mathrm{F}}$ for temporal fading is based on K-factors varying from $-12 \mathrm{~dB}$ to $-6 \mathrm{~dB}$, as proposed in [4] for large office environments. The calculation is done for 2.4 and $5.4 \mathrm{GHz}$ with the determined PL model (Tx position in front). We consider a coverage percentage of $90 \%$ to determine $\mathrm{M}_{\mathrm{S}}$. We assume an area $\mathrm{S}$ of $2,500 \mathrm{~m}^{2}$.

Based on this calculation, the influence of the different link parameters on \#AP, $\mathrm{P}$ and $\mathrm{TP}_{\max }$ is evaluated assuming a fixed range of $15 \mathrm{~m}$ (by tuning the transmit power) and 1 spatial stream (MCS 0 to 7). The results are summarized in Table II. Unless otherwise mentioned, the results apply to $2.4 \mathrm{GHz}$. A higher frequency gives a higher required $\mathrm{P}$, because the $\mathrm{PL}$ is approximately proportional to $1 / \lambda^{2}$ (see equation 5). Compared to SISO, MIMO $2 \times 2$ gives a lower required $\mathrm{P}$, due to a better (lower) sensitivity. Compared to a bandwidth of $20 \mathrm{MHz}, 40 \mathrm{MHz}$ requires a higher $\mathrm{P}$ (due to a worse sensitivity), but allows a higher $\mathrm{TP}_{\max }$. A higher (fixed) range requires of course less access points, but a lower $\mathrm{P}$ as well.
This is due to a PL exponent lower than 2, which results in a decreasing relation of $\mathrm{P}$ vs $\mathrm{P}_{\mathrm{T}}$ (see Fig. 5). Due to a $\mathrm{PL}$ exponent of 3.5, the TGn channel model predicts that a higher (fixed) range requires a higher $\mathrm{P}$.

The determined PL model predicts maximum ranges (i.e. for the maximum allowed transmit power) higher than $139 \mathrm{~m}$. Therefore, $\mathrm{TP}_{\max }$ is not influenced by the configuration, bandwidth or (fixed) range (see Table II). As mentioned before, the TGn channel model predicts lower maximum ranges, which can result in an influence on $\mathrm{TP}_{\max }$ for a (fixed) range from $40 \mathrm{~m}$.

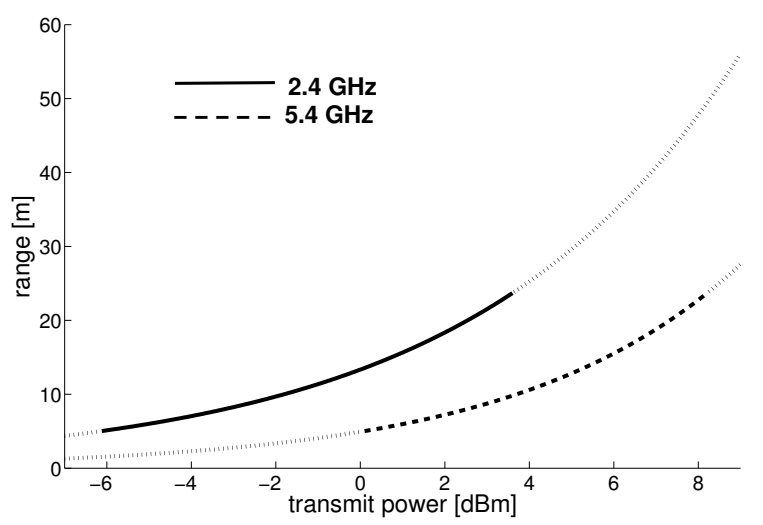

Fig. 3. Calculated range vs transmit power, based on the PL model, determined for a large conference room. The dotted line indicates that the range is out of the region where the PL model could be experimentally determined.

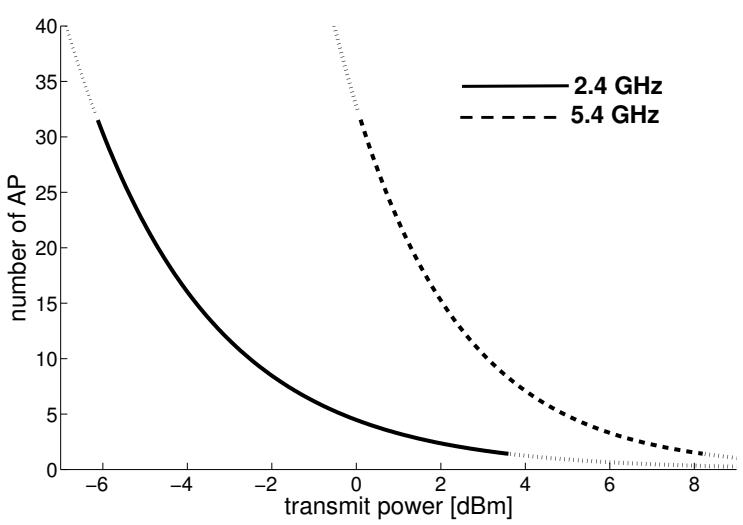

Fig. 4. Calculated number of access points vs transmit power, based on the PL model, determined for a large conference room. The dotted line indicates that the range is out of the region where the PL model could be experimentally determined.

\section{CONCLUSIONS}

We determined a PL model for 802.11n in large conference rooms, based on PL measurements. The PL could be described accurately by a one-slope model with one standard deviation. PL exponents varying from 1.2 to 1.7 were found.

Based on this PL model, the effect of frequency (2.4 vs $5 \mathrm{GHz}$ ), configuration (SISO vs MIMO (spatial diversity)), 


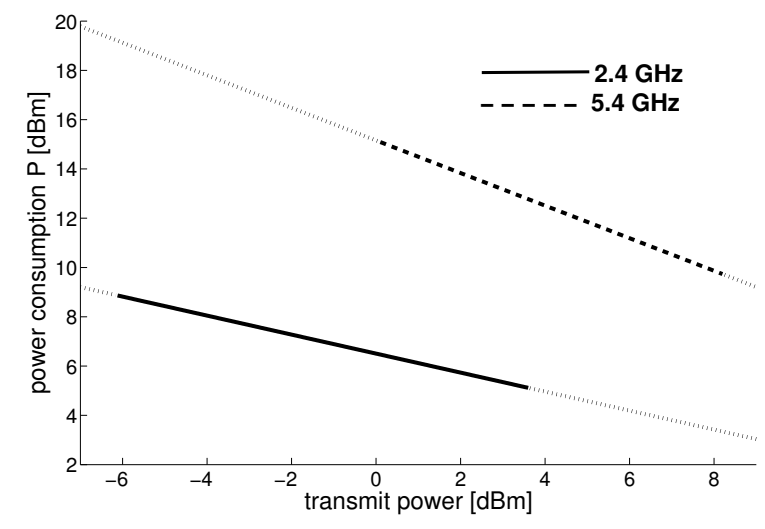

Fig. 5. Calculated total power consumption vs transmit power, based on the PL model, determined for a large conference room. The dotted line indicates that the range is out of the region where the PL model could be experimentally determined.

TABLE II

THE EFFECT OF DIFFERENT LINK PARAMETERS ON \#AP, TOTAL POWER CONSUMPTION (P) AND TP $\mathrm{TAX}_{\text {MAX }}$ THE INFLUENCE IS EVALUATED FOR A FIXED RANGE OF $15 \mathrm{M}$ (UNLESS OTHERWISE MENTIONED). THE EFFECT IS CALCULATED FOR THE 2 TX POSITIONS, WHICH CAN GIVE DIFFERENT VALUES, INDICATED BY (1) IN TABLE.

\begin{tabular}{|c|c|c|c|}
\cline { 2 - 4 } \multicolumn{1}{c|}{} & \#AP & $\mathrm{P}[\mathrm{mW}]$ & $\mathrm{TP}_{\max }[\mathrm{Mbps}]$ \\
\hline $\begin{array}{c}\text { frequency } \\
2.4 \rightarrow 5.4 \mathrm{GHz}\end{array}$ & $=$ & $\times 3.2-4.6^{(1)}$ & $65 \rightarrow 65$ \\
\hline $\begin{array}{c}\text { SISO } \rightarrow \\
\text { MIMO } 2 \times 2\end{array}$ & $=$ & $\times 0.25$ & $65 \rightarrow 65$ \\
\hline $\begin{array}{c}\text { bandwidth } \\
20 \rightarrow 40 \mathrm{MHz}\end{array}$ & $=$ & $\times 2$ & $65 \rightarrow 135$ \\
\hline $\begin{array}{c}\text { range } \\
15 \rightarrow 30 \mathrm{~m}\end{array}$ & $\times 0.25$ & $\times 0.5-0.8^{(1)}$ & $65 \rightarrow 65$ \\
\hline
\end{tabular}

bandwidth (20 vs $40 \mathrm{MHz}$ ) and transmit power on number of access points, total power consumption and possible (physical) throughputs has been investigated. Compared to SISO, MIMO is advantageous in every aspect. Compared to $2.4 \mathrm{GHz}, 5 \mathrm{GHz}$ is worse. A higher range (by tuning the transmit power) requires less access points, as well as a lower total power consumption, due to a PL exponent lower than 2.

\section{ACKNOWLEDGMENT}

This work was supported by the OMUS (Optimizing Multimedia Service Delivery) project, co-funded by the IBBT (Interdisciplinary institute for BroadBand Technology), a research institute founded by the Flemish Government in 2004, and the involved companies and institutions.

\section{REFERENCES}

[1] IEEE Std 802.11n ${ }^{T M}-2009$ Amendment 5 to Part 11: Wireless LAN Medium Access Control (MAC) and Physical Layer (PHY) Specifications: Enhancements for Higher Throughput, IEEE Std., Oct 2009.

[2] V. Erceg et al, “TGn channel models," IEEE, Tech. Rep., May 2004.

[3] D. De Zutter and F. Olyslager, Applied Electromagnetics. Ghent University, 2005
[4] R. J. C. Bultitude, "Measurement, characterization and modeling of indoor 800/900 MHz radio channels for digital communications," IEEE Commun. Mag., 1987. 